\title{
Control Strategies for a Multi-Legged Hoping Robot
}

\author{
Rolf A. Lüders, Dimi Apostolopoulos and David Wettergreen
}

\begin{abstract}
In this paper, we present locomotion control strategies for a novel multi-legged spherical hopping robot named "Robotic All-Terrain Surveyor" (RATS). The conceptual robot has a spherical body roughly the size of a soccer ball, with 12 legs equally distributed over its surface. While the 12legged spherical version of the robot is still on its design phase, we have experimented with a planar prototype of the robot to study the control problem in a simpler form. This work presents novel control strategies for the RATS planar robotic platform, which consist in the development of running and jumping gaits. Simulators were implemented as tools to help the development process. Physical experiments with the 5-legged device validate the control approaches and demonstrate the performance of the system and its potential for the future.
\end{abstract}

\section{INTRODUCTION}

The conceptual robot, named Robotic All-Terrain Surveyor (RATS), has a spherical body with 12 legs equally distributed over its surface. The legs are pneumatic linear actuators, which are oriented such that their axes of motion are normal to the surface of the sphere. The objective of this robot is to be able to circumvent complex obstacles and traps through rough terrain.

The RATS project is funded by BOEING Corporation and it is developed at Carnegie Mellon University. The objective is to develop a scout and/or surveyor robot for military or search \& rescue purposes. It must be able to negotiate with extreme terrains and operate for long distances.

The robot will operate on compressed $\mathrm{CO}_{2}$, which is stored on a central high-pressure chamber located at the center of the sphere. The spherical shape has the natural advantage that can allow the robot to roll downhill very efficiently. Also, it is inherently stable since it does not have an upright posture, and with its 12 legs it is very likely that at least one of them is in contact with a traction surface. Its hopping ability allows it to jump or hop over obstacles that other wheeled vehicles cannot.

The 12-legged spherical version of the robot is still on its design phase and no prototype has been built yet (by the time this was written). Instead, a simpler planar prototype of the robot is available to study the control problem. The planar robot consists of a wheel with 5 legs pointing radially, that rotates freely over the main axis of a radius arm that constraints its motion into a pseudo-plane. Fig. 2 shows a CAD drawing of the planar robot attached to the radius arm.

R. Lüders is with the Robotics Institute, NREC, Carnegie Mellon University, Pittsburgh, PA 15201, USA rluders@andrew. cmu. edu

D. Apostolopoulos is with Faculty of Robotics Institute, NREC, Carnegie Mellon University, Pittsburgh, PA 15201, USA dav1@ CS . cmu . edu

D. Wettergreen is with Faculty of Robotics Institute, FRC, Carnegie Mellon University, Pittsburgh, PA 15213, USA dsweri.cmu . edu

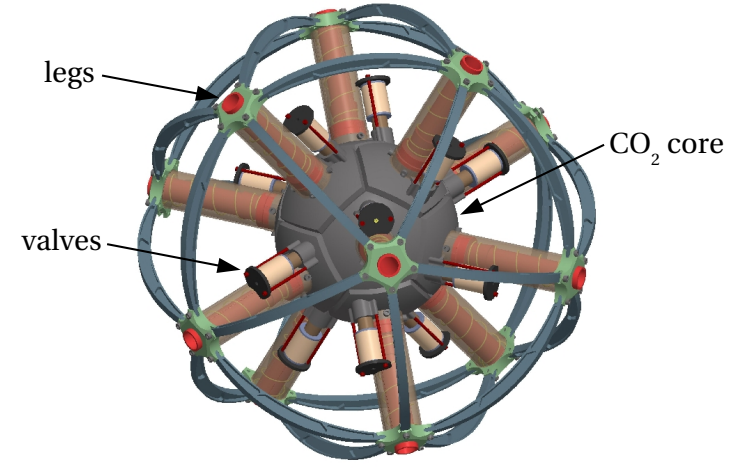

Fig. 1. CAD drawing of the spherical 3D RATS.

The air-cylinders run on compressed air which is supplied over a tether that goes through the radius arm.

This work presents novel control strategies for the RATS robotic platform that are based on dynamic locomotion. Two types of behaviors are analyzed for the planar robot: running, and jumping gaits. In both cases, successful controllers are found to achieve such behaviors.

\section{BACKGROUND}

The idea of creating a spherical robot that has extendable legs around its body seems to be very unique to RATS. However, others have developed systems that have some particular similarities with RATS. These robots include single-legged hoppers and spherical rolling robots.

\section{A. Single-legged Hoppers}

Single-legged robots can be categorized under: (1) continuous motion type, which are dynamically stabilized robots that can hop without stopping, and (2) discontinuous motion robots, that need a pause between jumps for reorientation and/or recharge. Continuous motion robots use active control systems to maintain their dynamic equilibrium and can hop in a periodic manner. One of the first attempts of singlelegged hoppers to use active stabilization was developed by K. Matsouka [8] using a high-force electric solenoid. Matsuoka's work was followed by M. Raibert [10] by creating a 2D single-legged hopper in 1980 that was constrained to a $2 \mathrm{D}$ pseudo-plane using a boom that rotates over a central axis. The robot used a double acting air cylinder [9]. Another milestone in single-legged dynamic locomotion is the bow leg hopping robot developed by Garth Zeglin and Ben Brown at $\mathrm{CMU}$ [3] [2]. 
Relating to discontinuous motion hopping robots, a JPL/Caltech group (Fiorini and Burdick [7], [5]) has developed several systems that use discrete leaps for locomotion. Their robots use a single-foot mechanism driven by releasing a compressed spring connected to the foot. After each jump, the spring is retracted with an electric motor. Another robot that uses a similar hopping mechanism was built at Sandia National Laboratories [13], that instead of using a spring as its thrust mechanism, it uses a piston driven by a combustion chamber.

\section{B. Spherical Rolling Robots}

Spherical rolling robots do not use legs or pistons for locomotion, they usually operate by changing the position of their center of mass to roll into a certain direction. Bicchi et al. [6] proposed a spherical robot that had a small car laying at the bottom of the sphere. Alternatively, Bhattacharya and Agrawal et al. [1] developed a spherical robot that had rotors inside to induce the motion of the sphere. Another interesting robot that falls into the same category is the "Gyrover" developed at the Robotics Institute of Carnegie Mellon University [4]. It is a single-wheel robot that uses an internal gyroscope for roll stabilization.

\section{Discussion}

The RATS configuration shares particular characteristics with the systems described above, e.g. it uses 12 pneumatic pistons for locomotion where each piston behaves similar to the ones on the single-legged machines. When all the pistons are inside, the robot can roll freely as the spherical robots described above.

Whether RATS belongs to the class of continuous or discontinuous motion systems, the answer is that it depends on the control mode used. For obstacle jumping it can leap from rest using a single foot and jump an obstacle, corresponding to the discontinuous motion category. If the robot is running in a predetermined direction by rolling and firing certain pistons sequentially, it can be assumed the robot is operating in a continuous motion regime.

\section{5-LEGGED PLANAR ROBOT DESIGN AND SIMULATION}

The 5-legged planar prototype of RATS is a novel design derived from the 3D 12-legged RATS concept. It is a simpler version of the $3 \mathrm{D}$ robot, which has proved to be extremely useful for devising and testing control strategies that could be extrapolated to the more complex 3D system. The planar robot has also been useful for the development and validation of mechanical parts such as the cylinder-piston mechanism.

\section{A. Mechanical Design}

In principle the planar robot is a disc containing five pneumatic actuated legs oriented radially outwards, the disc rotates over an unactuated axle and is constrained into a vertical plane of motion by a radius arm (See Fig. 7). The wheel is connected to the articulated radius arm (boom) which holds it in alignment and allows rotation in a vertical

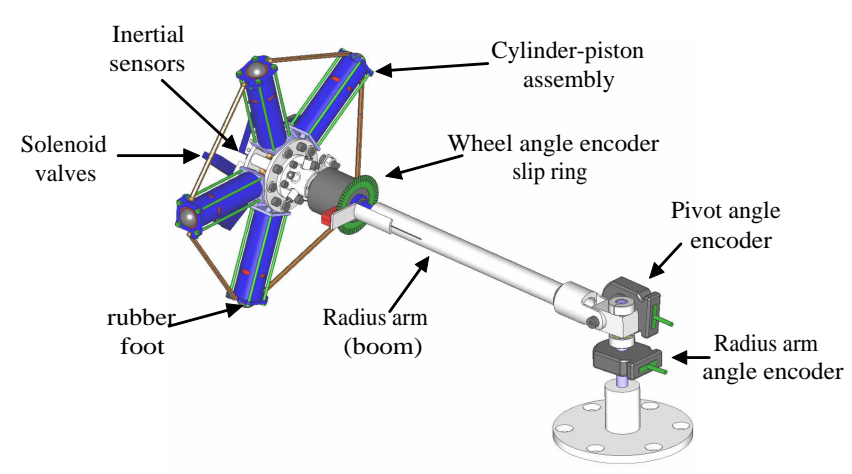

Fig. 2. Planar robot mechanical description diagram.

pseudo-plane. Fig. 2 shows a CAD design diagram that describes the general setup. The actuation is given by five air cylinders powered by compressed air that is available through a tether. The air flow is administered to the cylinders by 5 solenoid air valves mounted on the disc. There is a slip ring mechanism to by-pass the electrical connections and the air supply.

\section{B. Sensing}

Several sensors are attached to the robot, these include three encoders to measure the radius, pivot, and wheel angles; a MEMS gyroscopes to measure the wheel angular velocity; and two 3-axis accelerometers to measure the accelerations of the wheel (see Fig. 2). The input and actuation signals are managed by a multifunction input/output (MIO) board which is interfaced to the main PC computer via the PCI bus.

\section{Simulation}

A complete computer simulator of the planar robot was implemented to test new control strategies. This proved to be particularly useful to speed up the process of testing different control strategies and to understand better the dynamics of the system. The physics engine SDK used was Open Dynamics Engine (ODE) [12]. The package includes advanced features such as several built-in joint types and integrated collision detection with friction. One of the effects that could not be directly simulated with the physics engine was the force generated by the pneumatic cylinders. To take this in effect, a non-linear pressure dynamics model of the complete pneumatic system was fitted. The model considered the air compressibility through the chambers; the non-linear flow through the valve; and the piston friction with the wall of the cylinder. The pneumatic model was later integrated with the main ODE simulator.

To fit the model a special benchtop single-legged system was used. The system replicated one of the 5-legged air cylinders and had extra sensors to acquire information such as the pressure inside the chamber and position of the piston.

The non-linear model is based on the work from Richie and Hurmuzlu in [11]. The model takes in consideration the air compressibility; non-linear air flow through the valve; and 


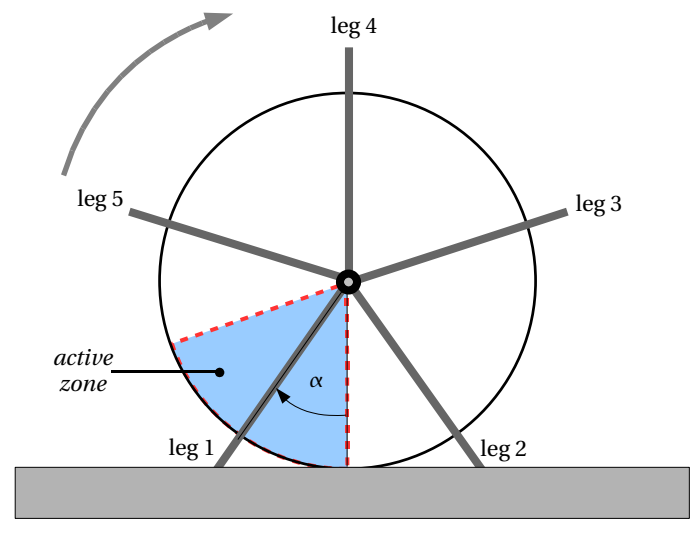

Fig. 3. Description of the main parameters of the running gait. The shaded zone corresponds to the active zone. The $\alpha$ angle is defined between the normal of the surface and the leg (any of the 5) that is inside the active zone. Each leg is fired at a predetermined $\alpha=\alpha_{f}$ once it enters the fixed active zone.

the air line delay and attenuation among others. A multivariable unconstrained non-linear optimizer that operated with a single error metric was used for finding the hidden parameters.

\section{PLANAR ROBOT CONTROL GAITS}

The key complication regarding the controllability of RATS is the lack of control over the angle of a certain leg and the ground. Other robots like Raibert's single-legged continuous hoppers [10] use a lightweight leg that can be servoed to arbitrary angles when the robot is in its flightphase. In RATS, all legs are fixed to the center wheel and it is not possible to actuate directly over the pose of them. Nevertheless, the fact that the wheel can rotate, can allow the legs to be positioned sequentially at a desired angle with respect the ground.

\section{A. Running}

The running gait is based on the rolling motion of the wheel. The legs are fired sequentially as they make contact with the ground surface at a determined angle such that they apply horizontal and vertical forces that induce the rotation of the wheel.

To explain the basic running controller we define a sector of the wheel, which we call active zone, that translates but does not rotate with the wheel as shown in Fig. 3. We also define a variable angle alpha $(\alpha)$ that represents the angle between the normal of the ground surface and the leg that is inside the active zone. We finally define the parameter alpha-fire $\left(\alpha_{f}\right)$ as the angle $\alpha$ at which the leg is fired after it enters the active zone when the wheel is rotating.

For the robot to run as intended, $\alpha_{f}$ needs to be carefully chosen, since firing the leg too early (small $\alpha_{f}$ 's) will make the robot to hop vertically and firing too late will cause part of the stroke to be lost in the air because of the advance of the wheel (rotation.)

The action of firing a leg consists in opening the pneumatic valve for a predefined amount of time. We call this parameter

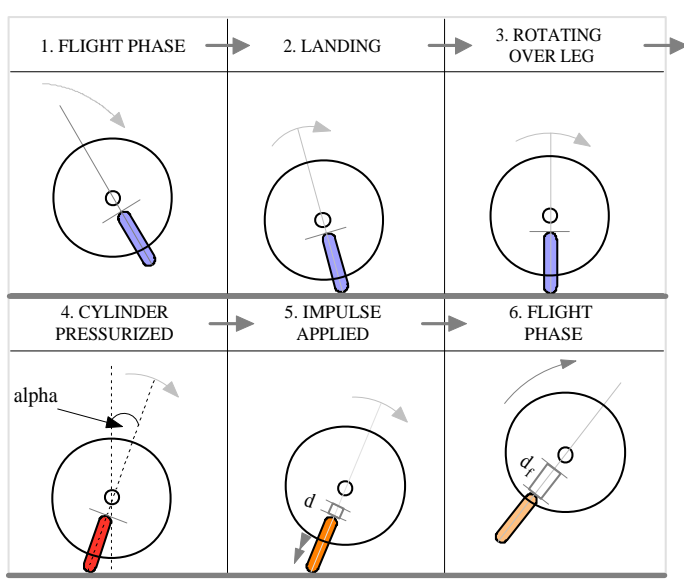

Fig. 4. Landing (stance-phase) and takeoff (flight-phase) of the robot on one leg sequence for jumping

valve-opening-time $\left(t_{v}\right)$. The longer the valve remains open, the longer the the power stroke will be (till it reaches its mechanical limit.)

This low-level gait is then governed just by two parameters, the $\alpha_{f}$ angle the $t_{v}$ time interval. The sequential firing of the legs allows to continuously inject energy that impulses the wheel to rotate and advance. This concept was initially tried in simulation, an animation can be seen at: ADD REF TO ANIMATION.

\section{B. Jumping}

In this section the jumping strategy is presented, which consists in controlling the robot to allow it to jump over obstacles, or steps. The jumping sequence consists in first acquiring horizontal momentum by running using the running strategy presented above. Once the robot is near the obstacle, it lands on one leg which acts as a hinge joint with the ground, and at a predefined angle the leg is fired to achieve a trajectory in the air to jump over the obstacle. Fig. 4 summarizes the sequence of events for jumping.

1) Dynamics Model: To analyze the interaction between the leg and the ground surface when jumping, a simplified dynamics model was derived using Lagrangian dynamics. The objective of this model is to understand better the resultant trajectory of the planar robot after a single leg has been fired at a certain angle $\alpha_{f}$ with respect the normal of the ground surface. The trajectory found can be used to determine the optimal $\alpha_{f}$ for hopping over a known obstacle. The equations assume that robot first lands on a single leg generating a hard contact with the ground which acts as a hinge joint.

Fig. 5 shows a free-body diagram of the system analyzed, where $\alpha$ (" $a$ " in the figure) is the angle between the leg and the ground surface, $R$ is the the radius distance between the center of the disk and the contact point. $R$ is divided in two parts, $r_{s}$ is the radius of the wheel and $d$ is the extension distance of the leg. The disk is modeled as thin disk with mass $m$, and the leg is assumed to be massless.

To determine the dynamics of the system when a force 


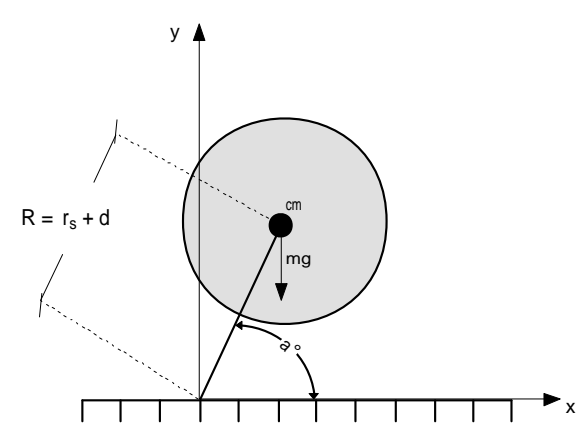

Fig. 5. Free-body diagram of the robot with one leg in contact with the ground plane

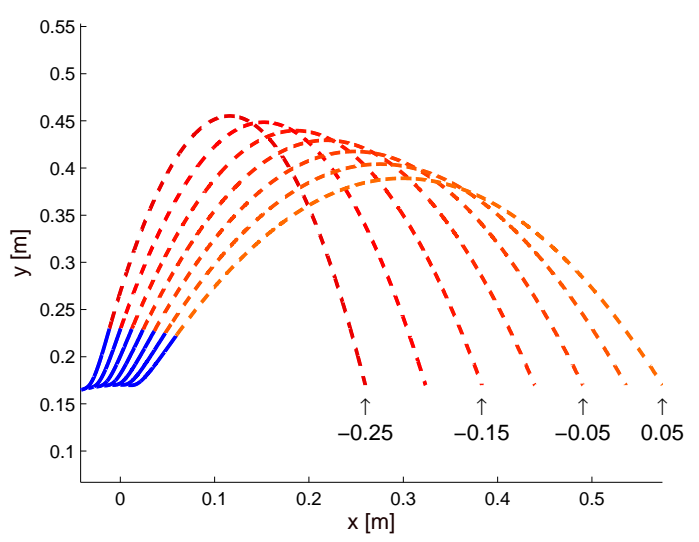

Fig. 6. Family of trajectories of the center of the robot under different firing angles. Each trajectory has a stance phase (solid line) and a flight phase (dotted line). The number below each trajectory is the firing angle $\alpha_{f}$.

is applied by the leg, the Lagrangian equations were used. $T$ is the kinetic energy (Eq. 1) and $U$ the potential energy (Eq. 2). The generalized coordinates are: the piston travel " $d$ " and the angle between the the ground plane and center of the disk with respect the contact named " $\alpha$ ".

$$
\begin{gathered}
U=m g(d+R) \sin (\alpha) \\
T=\frac{1}{2} I \dot{\alpha}^{2}+\frac{1}{2} m \dot{\alpha}_{i}^{2}(d+R)^{2}+\frac{1}{2} m \dot{d}^{2}
\end{gathered}
$$

Solving for $\ddot{\alpha}$ and $\ddot{d}$ after applying the equations of Lagrange, the resultant accelerations of the generalized coordinates are given by Eqs. 3 and 4 respectively.

$$
\begin{gathered}
\ddot{\alpha}=\frac{-m g(d+R) \cos (\alpha)-2 m \dot{\alpha} \dot{d}(d+R)}{I+m(d+R)^{2}} \\
\ddot{d}=\frac{F+m \dot{\alpha}^{2}(d+R)-m g \sin (\alpha)}{m}
\end{gathered}
$$

2) Model Analysis: The equations were integrated using different initial angular velocities $(\dot{\alpha})$ and firing angles $\alpha_{f}$. If the piston reaches the maximum displacement $(0.06 \mathrm{~m})$ the robot is assumed to be in flight phase and the departing angular and linear velocities are used to simulate a rigid body

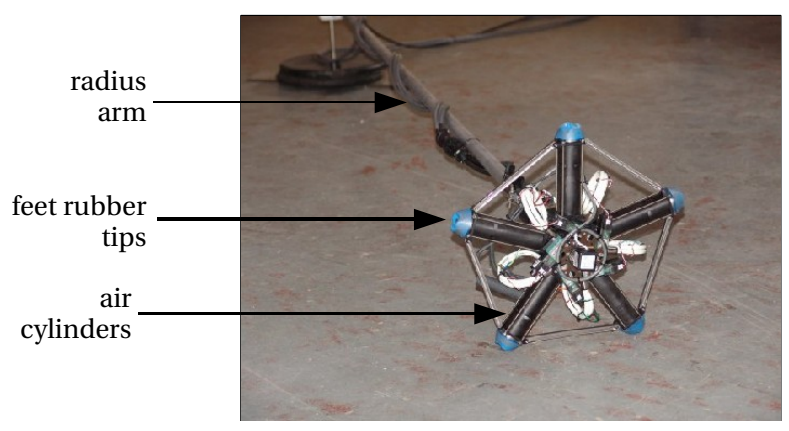

Fig. 7. 5-legged planar robot prototype image and description.

with no constraints (ballistic motion). The force applied was obtained from the pressure dynamics model found.

Fig. 6 shows a family of trajectories of the center of the wheel under different firing angles $\left(\alpha_{f}\right)$. The contact phase (leg touching the ground) is represented by the solid lines and the flight phase by the dotted lines. The approaching angular velocity of the wheel was set at $\dot{\alpha}=-5.0(\mathrm{rad} / \mathrm{s})$. The firing angles range from $\alpha_{f}:-0.25(\mathrm{rad})$ to $0.05(\mathrm{rad})$. The plot clearly shows that the later the leg is fired (larger $\alpha_{f}$ ) the greater is the distance travelled in the horizontal direction and the least in the vertical direction.

\section{IMPLEMENTATION AND RESULTS}

The results and implementation details of the controllers are explained in this section. All tests were performed using the 5 legged planar robot. An illustration of the experimental setup is shown in Fig 7.

\section{A. Running Controller}

1) Manual Operation: The first running controller was implemented in such a way that the operator could manually control the parameters $\left\{\alpha_{f}, t_{v}\right\}$ through the use of the keyboard of the controller PC in real time while the robot was moving. The wheel angle was the only state variable used to control the robot, and it was used to calculate the $\alpha$ angle. It was found that starting with a large $\alpha_{f}$ (late firing), the robot would start moving by its own, and that if the $\alpha_{f}$ was reduced once it had gained momentum, the robot would run fast and smooth. ADD VIDEO REF

2) Open-Loop Controller: Instead of having a human operator controlling the $\left\{\alpha_{f}, t_{v}\right\}$ parameters, we formulated a controller that automatically varies the $\alpha_{f}$ angle to accelerate fast from rest without intervention. The controller obtains the $\alpha_{f}$ value in an open-loop mode by evaluating a function with respect to time. An arbitrarily chosen smooth function was used to transition from the initial alpha-fire $\left(\alpha_{\mathrm{fi}}\right)$ to the final alpha-fire $\left(\alpha_{\mathrm{ff}}\right)$. The constants $\alpha_{\mathrm{fi}}$ and $\alpha_{\mathrm{ff}}$ were found experimentally with the manual controller presented before. $\alpha_{\mathrm{fi}}$ makes the robot to start reliably from rest, and $\alpha_{\mathrm{ff}}$ causes the robot to run fast and smooth. The function used is given by the following Eq. 5 .

$$
\alpha_{f}(t)=A \tanh (B t-C)+D
$$




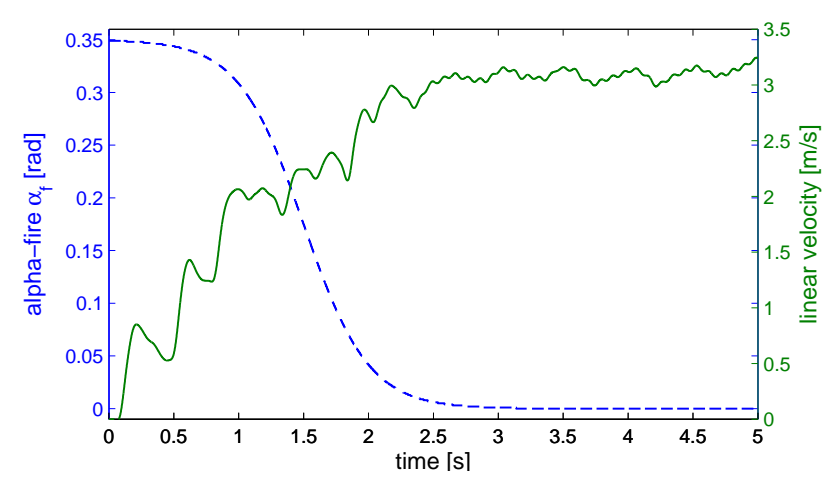

Fig. 8. Accelerating from rest using the open-loop control results. The plot shows the $\alpha_{f}$ (dotted line) and the linear velocity (solid line) versus time.

This particular function was selected because it does a smooth transition and it is highly customizable through few parameters. The parameters $A$ and $D$ can be found given that $\alpha_{f}\left(t_{0}\right)=\alpha_{\mathrm{fi}}$ and $\alpha_{f}\left(t_{f}\right)=\alpha_{\mathrm{ff}}$ then,

$$
\begin{aligned}
& A=\frac{\alpha_{\mathrm{ff}}-\alpha_{\mathrm{fi}}}{2} \\
& D=\frac{\alpha_{\mathrm{ff}}+\alpha_{\mathrm{fi}}}{2}
\end{aligned}
$$

,where $B$ is the slope of the transition, and $C$ is the lapse of time before the center of the transition occurs. By using the simulator the parameters $B$ and $C$ were tuned for fast acceleration. The controller was then tested in the actual robot and it worked adequately. The parameters found after a brief tunning were: $B=2.1(\mathrm{rad} / \mathrm{s})$, and $C=3.0(\mathrm{~s})$. A constant $t_{v}$ time of $40(\mathrm{~m} / \mathrm{s})$ was used for this experiment.

Fig. 8 shows that the wheel accelerates from rest to $3.2(\mathrm{~m} / \mathrm{s})$ in less than 3 seconds.

3) Closed-loop Controller: One of the main drawbacks of varying the $\alpha_{f}$ in an open-loop mode is that is not robust against perturbations. The controller presented next uses the fact that the parameter $\alpha_{f}$ is highly related to the angular rate of the wheel $(\omega)$ for accelerating, this means the faster the wheel rotates, the earliest the leg needs to be fired. This allows the maximum force point of the piston to be applied when the leg is inside the active zone at a predefined optimal angle. This accounts for the delays of the valve to open and of the maximum pressure to build, which is very similar to the ignition timing system in a gasoline internal combustion engine.

A function was used to relate the angular velocity to the $\alpha_{f}$ angle for closed-loop operation. This function was made of two linear functions concatenated since it was determined experimentally that the $\alpha_{f}$ needs to be decreased at a lower rate when the robot is starting than when the wheel is rotating faster.

$$
\alpha_{f}(\omega)=\left\{\begin{array}{lll}
C \omega+D & \text { if } & \omega<\omega_{s} \\
E \omega+F & \text { if } & \omega \geq \omega_{s}
\end{array}\right\}
$$
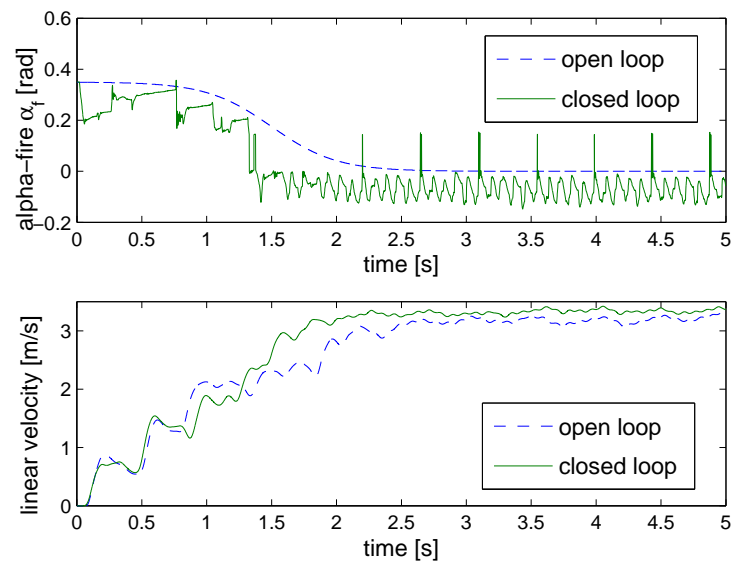

Fig. 9. Accelerating from rest using the closed-loop controller. Results of the close-loop controller (solid line) are compared with ones of the openloop controller (dotted line)

with,

$$
\begin{gathered}
D=\alpha_{\mathbf{f i}} \\
E=\frac{\alpha_{\mathbf{f f}}-\alpha_{\mathbf{f i}}}{\omega_{\mathbf{f}}} \\
F=\omega C+D
\end{gathered}
$$

$\omega_{s}$ is the switching angular velocity, and was found experimentally by trying different values, the same for $C$, the slope of the starting linear function. This approach of using two linear functions is an approximation of the non-linear function that governs the optimal relation between $\omega_{s}$ and $\alpha_{f}$. Learning techniques could be used to find such relation, which is proposed as future work.

Fig. 9 shows the results of the close-loop controller with the robot. The close-loop controller actually outperformed the open-loop controller, converging to slightly negative $\alpha_{f}$ 's. This means the valves were being energized slightly before the correspondent leg was normal to the ground surface. To allow this, the active zone was rotated slightly counterclockwise to allow for negative $\alpha_{f}$ angles. The angular velocity of the wheel $(\omega)$ was acquired using the MEMS gyroscopes on board. The sensor data was filtered using an IIR filter in realtime to filter noise that was not related to the primary rotation mode of the wheel.

4) Initialization: Since the wheel angle sensor was a relative angle encoder, the MEMS accelerometer was used to measure the gravity vector and thus determine automatically which two feet were in contact with the ground surface every time the algorithm was initialized.

\section{B. Jumping controller}

To test the jumping concept a preliminary jumping controller was implemented for the 5-legged robot. The strategy consisted in accelerating using the first three legs using the running mode presented above to acquire horizontal momentum. Once the third leg is fired the controller is switched into the jumping control mentioned above using 
the fourth leg to jump. The transition occurs very naturally because the actuation is very discrete between legs, therefore switching controllers between firings does not disturb the behavior of the robot.

A problem found was that the horizontal velocity prior to the jump was not repeatable. The most probable causes are changes in the friction between the foot and the floor, and non-predictable behavior of the air cylinders. As a consequence, the initial angular velocity $\dot{\alpha}$ of the jumping leg was not always the same, therefore using a constant $\alpha_{f}$ generates unpredictable flight trajectories. To overcome this problem, the Lagrangian dynamic model of the jumping motion was used to find a relation that would retard the firing of the leg if the approach speed was low, and advance if it was fast, so that the distance covered by the jump would remain relatively constant.

The controller implemented was designed to jump over a constant horizontal distance of 1 meter regardless the approaching speed. If the robot approaches at a high speed then most of the propulsion is used to gain height by using a small $\alpha_{f}$. If the robot approaches slow then a large $\alpha_{f}$ is used to direct most of the power horizontally and reach the target proposed.

To search for the relation between the initial $\dot{\alpha}$ and the $\alpha_{f}$ for our goal, the model was initialized with different initial $\dot{\alpha}$ 's (in the expected range seen from the behavior of the robot). For each initial $\dot{\alpha}$ the $\alpha_{f}$ was varied and the jump was simulated. The $\alpha_{f}$ was adjusted till it generated a jump that covered the 1 meter distance within a small error margin. This was done for 5 different initial $\dot{\alpha}$ 's, and the results proved that the relation was very linear. The equation of the relation is,

$$
\alpha_{f}=A \dot{\alpha}_{i}+B
$$

The values found were $A=-0.1(s)$ and $B=0.46(\mathrm{rad})$.

Fig. 10 shows the results of the robot jumping over a cardboard box with a squared profile of $21(\mathrm{~cm}) x 21(\mathrm{~cm})$ without touching it using the variable $\alpha_{f}$ scheme. The left plot is the position of the center of the wheel, and the right plot shows the valve signals, both plots present the complete jumping sequence from rest (note that the $\mathrm{x}$-scale differs from the y-scale in the position plot). A video of this result can be found at: ADD VIDEO REF

\section{CONCLUSION}

The novel 12-legged RATS concept diverges radically from traditional mobile robots, therefore the problem of the controls and sensing is still in its very early development

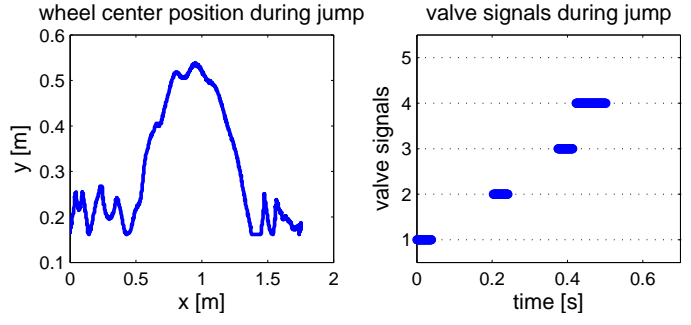

Fig. 10. Left plot: Position of the center of the wheel during the jump. Right plot: Valve signals versus time during the jump. The y-axis labels represent the valve number. The marked zones indicate the valve was energized at that time.

stages. This work proves some basic concepts on the 5legged prototype that could be used to control the 12-legged robot. Some of these concepts are the idea that the robot can sequentially fire the legs for running using the rolling motion of the body, and the fact that a single leg can be used for jumping if the body has enough approaching horizontal momentum.

\section{REFERENCES}

[1] Shourov Bhattacharya and Sunil Kumar Agrawal. Design, experiments and motion planning of a spherical rolling robot. In ICRA, pages 1207-1212. IEEE, 2000.

[2] Ben Brown and Garth Zeglin. The bow leg hopping robot. In ICRA, pages 781-786, 1998.

[3] Ben Brown and Garth Zeglin. Control of a bow leg hopping robot. In ICRA, pages 793-798, 1998.

[4] H. Benjamin Brown and Yangsheng Xu. A single-wheel, gyroscopically stabilized robot. IEEE Robotics and Automation Magazine, 4(3):39-44, September 1997.

[5] Joel Burkick and Paolo Fiorini. Minimalist jumping robots for celestial exploration. I. J. Robotic Res., 22(7-8):653-666, 2003.

[6] Centro e. Piaggio, Antonio Bicchi, Andrea Balluchi, Domenico Prattichizzo, and Andrea Gorelli. Introducing the "SPHERICLE": an experimental testbed for research and teaching in nonholonomy, 1997. Warning: the year was guessed out of the URL.

[7] Paolo Fiorini and Joel W. Burdick. The development of hopping capabilities for small robots. Auton. Robots, 14(2-3):239-254, 2003.

[8] K. Matsuoka. A mechanical model of repetitive hopping movements. Biomechanisms, 5:251-8, 1980.

[9] Marc Raibert, H. Benjamin Brown, Michael A. Chepponis, Eugene F. Hastings, Sesh Murthy, and Frank C. Wimberly. Dynamically stable legged locomotion: Second report to darpa, october 1,1981 - december 31,1982. Technical Report CMU-RI-TR-83-01, Robotics Institute, Carnegie Mellon University, Pittsburgh, PA, January 1983.

[10] Marc H. Raibert. Legged Robots That Balance. MIT Press, Cambridge, MA, 1986.

[11] Edmond Riche and Yildirim Hurmuzlu. A high performance pneumatic force actuator system part 1 - nonlinear mathematical model. Transactions ASME, 2000.

[12] Rusell Smith. Open Dynamics Engine v0.5 User Guide, Frebruary 2006. www.ode.org.

[13] Peter Weiss. Hop...hop...hopbots! Science News, 159(6):88, Feb. 10 2001. 